Atif Naveed 155

\title{
Stock Market Development and Financial Intermediary Development in Pakistan (1991-2001)
}

\section{Atif Naveed ${ }^{*}$}

A healthy financial sector is an essential ingredient for a strong and prosperous economy as it performs the important functions of mobilising and allocating savings to meet the funding requirements of business and industry. This in turn enables the commercial and industrial base to expand leading to higher economic activity with increased levels of output and employment.

The above stated roles are performed to perfection in the developed countries where a vast web of financial institutions and financial instruments exist to channelise savings into investments. Moreover, the developed financial markets have a very effective distributive system whereby the investors reap the benefits of their investments according to set distributive rules. As against this developing economies lack an efficient financial system. This has invited many cross country and time series studies to gauge the level of financial development in the developed and developing economies.

Studies show that large stock markets tend to be less volatile, more liquid and less concentrated in a few stocks than smaller markets (e.g. Demurigic Kunt and Ross Levine 1996). Moreover, countries that liberalise restrictions on capital and dividend flows show a marked improvement in the functioning of their stock exchanges (e.g. Levine and Sara Zervos 1995). Maksimovic and Kunt (1996) have worked on the interaction between stock market and financial intermediaries and through cross-country analysis confirmed that stock market development moves together with the development of financial intermediaries. Considerable research on the stock market and banking sector of Pakistan has also been undertaken. Khilji (1993) has observed the behaviour of stock returns in the emerging market of Pakistan and surprisingly found that investors in the Pakistani stock market having diversified portfolios are subject to the same amount of risk as investors with one industry portfolios. Salman Shah (1989) has discussed the contribution of

\footnotetext{
* The author is Research Officer, State Bank of Pakistan, Karachi.

The author is grateful to Dr. Muhammad Naeem (Asst. Prof. IBA) and Mr. Moinuddin (SBP Research Dept.) for their valuable comments that helped in the improvement of this paper.
} 
commercial banks, DFIs, investment banks and the equity market in financing medium and long term requirements.

The current study focuses on financial sector development in the context of Pakistan. In this paper, with time-series analysis, the development of the Pakistani stock market and financial intermediary sector over a period of 11 years (1991-2001) has been observed on a quarterly basis. Different indicators of stock market development have been constructed; namely stock market size, liquidity and market integration which reflect the level of development of the market from various angles. Similarly, several indicators of the financial intermediary sector have been constructed through which an attempt has been made to see the level of development of different sectors of financial intermediation namely the scheduled banking sector, development finance institutions (NBFIs), and saving schemes.

Since no single measure covers all aspects of the stock market and financial intermediary development therefore, aggregate indexes of the stock market and the financial intermediary sector are constructed that aggregate information on different indicators of the stock and financial intermediary sector. This enables us to get an idea about the overall level of development of the stock and financial intermediary sectors of Pakistan.

On the basis of the individual indicators and aggregate indexes, a correlation study of the stock market and financial intermediaries is carried out. Firstly, an attempt has been made to see whether the individual indicators of the stock market are correlated in an expected manner or not. Similarly, the correlation in the aggregate indexes of the stock market is tested. This exercise is carried out for the financial intermediary sector as well, both at the level of individual indicators and their aggregate indexes. Strong correlations between the individual indicators and indexes of the stock market and financial intermediary sector speak of how integrated the development has been in the two areas over the period of study.

Secondly, as debt and equity are frequently viewed as alternative sources of financing, an attempt has been made to empirically see if the two sectors support each other or there exists a trade-off between them. In this regard, correlations between the individual indicators of the stock market and financial intermediary sectors as well as their aggregate indexes are carried out.

This study is different from previous studies on certain grounds: Firstly, as regards Pakistan very little research has come forward on the development of both the stock market and financial intermediaries on a 
quarterly basis over an 11 year period. Only specific sectors of the stock market or financial intermediary channels have been researched with no such framework established which collectively, via various indexes, measures the development of the financial sector. Secondly, in cross sectional analysis, vastly different countries appear which may at times be sufficiently different to warrant separate analysis. While averaging over long periods during such studies many events such as changes in government policies, business cycles etc. which are important for the respective countries, may remain blurred. This study is free of such shortcomings.

\section{Methodology for the Construction of Indicators and Indexes}

Most of the indicators used for representing development in various sectors of the stock market and financial intermediaries are obtained by dividing the values of the indicators by nominal GDP to get a ratio of those indicators to $\mathrm{GDP}^{2}$. Thus we get relative measures of these indicators which have greater advantage in statistical analysis than their absolute values as they represent the values of these indicators with respect to the indicator of economic development i.e. GDP. The only one exception is the Turnover ratio which in itself is a ratio not involving GDP. All the individual indicators are constructed for both the end- period values as well as for the average values of the indicators. Thus we obtain two series for each individual indicator.

To produce an assessment of the overall level of capital market development, we construct means- removed indexes of the indicators. To construct means-removed indexes a two-step procedure is followed. Firstly, we obtain the means-removed values of all those indicators whose collective index is being constructed. The means-removed values of each indicator for each year equals the crude value of the indicator for a specific year ' $i$ ' minus the mean value of that indicator for all years divided by the absolute mean value for all years. To represent it more formally let $S(i, j)$ equal the value of the indicator ' $\mathfrak{j}$ ' for the year ' $i$ ' and $S(i)$ equal the average (mean) value of the indicator for all years, then the mean-removed value of the indicator ' $j$ ' i.e. $s(i, j)$ will be given by

$$
s(\mathbf{i}, \mathbf{j})=\frac{[S(\mathbf{i}, \mathbf{j})-S(\mathbf{j})]}{[\operatorname{Abs} S(\mathbf{j})]}
$$

This procedure is applied to all other indicators included within an index. Once the means-removed values for all indicators included in the

\footnotetext{
${ }^{2}$ The only authentic work on the quarterly GDP of Pakistan has been done by Kaiser Bengali as part of his Ph.D thesis. We use the real quarterly GDP values of Kaiser Bengali and convert them into nominal quarterly GDP.
} 
index are obtained, in the second step we take a simple average of the mean-removed values of the indictors to arrive at the value of the index for that year. All the indexes are constructed using both the end- period values as well as the average values of the individual indicators. Thus we obtain two series for each individual index.

This method of construction of indexes is repeated for all years and for all the indexes constructed in the study. The approach for the construction of means-removed indexes has been devised by Demirgic-Kunt and Ross Levine who work with the World Bank Policy Research Department and is a recognised approach for the construction of the indexes of capital market development.

\section{Indicators to Gauge Stock Market Development}

Conceptually, the level of stock market development is depicted in the form of progress in various sectors of the stock market namely, stock market size, liquidity, integration, volatility and market concentration. We in this paper, focus on market size, liquidity and market integration.

\section{Stock Market Size}

To measure the size of the market the market capitalisation ratio is used which is defined as the total value of the listed shares divided by the GDP (see Table 1). In order to arrive at the total value of the listed shares the total number of listed shares in the stock market is multiplied with the market price of the shares. In terms of the economic significance, the assumption behind market capitalisation is that it is positively correlated with the ability to mobilise capital and diversify risk.

\section{Liquidity}

Liquidity as defined by stock market analysts refers to the ability to easily buy and sell securities. Liquidity is an important attribute of stock market development because theoretically liquid markets improve the allocation of capital and enhance the prospects of the long-term commitment of capital. The dilemma with many profitable investments is that they require a long-term commitment of capital. However savers are often reluctant to relinquish control of their savings for long periods. Liquid equity markets help solve the problem by making the investment less risky and more attractive by allowing savers to acquire an asset-equity and to sell it quickly if they need access to their savings or want to alter their portfolios. At the same time, companies enjoy permanent access to capital raised through equity issues. 
Two measures of liquidity have been constructed. The first is total value traded to GDP, which equals the total shares traded divided by GDP (see Table 1). This ratio measures the organised trading of equities as a share of national output. Although the market capitalisation ratio may be large, there may be little trading. A second measure of liquidity is the Turnover ratio. Turnover ratio equals the value of total shares traded divided by market capitalisation (see Table 1). The Turnover ratio complements total value traded to GDP. The total value traded to GDP captures the trading compared with the size of the economy while the Turnover measures trading relative to the size of the stock market. Incorporating the information on total value traded to GDP and the Turnover ratio provides a more comprehensive picture of development of the stock market with respect to liquidity than a single indicator only.

\section{Market Integration}

A lot of importance is given to measuring the degree of integration of national stock markets and the world market. According to this indicator, Markets which are more integrated and which price risk more efficiently are more integrated. Under the maintained hypothesis, greater values of APT and ICAPM pricing errors reflect less international integration. We formulate two indicators of ICAPM pricing errors as measures of market integration. Firstly, we regress the SBP general price index for Pakistan's stock market on the Standard and Poor's ( $\&$ \& ) Global 500 index which is taken as a proxy for the global stock index. In this regard, we initially check for the stationarity of the two series and thereafter apply the Johansen Cointegration test to see if the SBP index is co-integrated with S\&P Global 500 Index. After verifying co-integration ${ }^{3}$, we take the residuals of regression results as ICAPM pricing errors. As large pricing errors reflect less market integration, we therefore multiply each pricing error with " -1 " (Table 1).

\footnotetext{
${ }^{3}$ The co-integration gets verified if the values of SBP index and S \& P index are taken up to $19981^{\text {st }}$ quarter and not if the values are taken for the entire period of study. This is so because the nuclear tests conducted by Pakistan in May 1998 depressed the stock prices and stock indexes in Pakistan badly while no such shocks occurred with US stock prices or S \& $\mathrm{P}$ Global 500 Index. If this distortion would not have been there, co-integration would have been most likely verified for the entire period. Taking this into account, we assume cointegration between the variables for the entire period and therefore compute pricing errors.
} 
Table 1: Indicators of Stock Market Development

\begin{tabular}{|c|c|c|c|c|c|c|c|}
\hline Year & $\begin{array}{l}\text { Mkt.Cap } \\
\text { /GDP }\end{array}$ & $\begin{array}{l}\text { Value } \\
\text { traded)/ } \\
\text { GDP }\end{array}$ & $\begin{array}{l}\text { Turn } \\
\text { over } \\
\text { ratio }\end{array}$ & $\begin{array}{l}\text { P.errors* } \\
\text { SBP-SP }\end{array}$ & $\begin{array}{l}\text { P.errors* } \\
\text { PAK- } \\
\text { USA }\end{array}$ & $\begin{array}{l}\text { Mkt.Cap } \\
\text { /GDP } \\
\text { (AV.Value) }\end{array}$ & $\begin{array}{l}\text { Turn } \\
\text { over ratio } \\
\text { (Av.value) }\end{array}$ \\
\hline Sep-90 & 0.077397 & 0.001705 & 0.022026 & -100.257 & -40.0982 & 0.076805 & 0.022195 \\
\hline Dec-90 & 0.078374 & 0.001521 & 0.019405 & -95.112 & -45.9081 & 0.078513 & 0.019371 \\
\hline Mar-91 & 0.07977 & 0.002101 & 0.026341 & -87.7726 & -4.62707 & 0.076514 & 0.027462 \\
\hline Jun-91 & 0.092759 & 0.003181 & 0.034291 & -70.0479 & -6.00675 & 0.088963 & 0.035754 \\
\hline Sep-91 & 0.114082 & 0.004659 & 0.040842 & -42.5416 & -36.8533 & 0.112662 & 0.041357 \\
\hline Dec-91 & 0.183579 & 0.006295 & 0.034291 & -13.1878 & -22.9988 & 0.149923 & 0.041989 \\
\hline Mar-92 & 0.176517 & 0.005994 & 0.033956 & -3.88714 & -12.7464 & 0.181641 & 0.032998 \\
\hline Jun-92 & 0.194819 & 0.004998 & 0.025656 & -10.2968 & -8.83347 & 0.190061 & 0.026298 \\
\hline Sep-92 & 0.176794 & 0.006037 & 0.034145 & -16.4585 & -22.5114 & 0.173752 & 0.034742 \\
\hline Dec-92 & 0.181525 & 0.005631 & 0.031022 & -12.066 & -21.8388 & 0.178552 & 0.031539 \\
\hline Mar-93 & 0.165388 & 0.004938 & 0.029855 & -22.7878 & -22.7987 & 0.172868 & 0.028563 \\
\hline Jun-93 & 0.160565 & 0.00718 & 0.044716 & -13.8737 & -25.8129 & 0.160088 & 0.044849 \\
\hline Sep-93 & 0.18417 & 0.009115 & 0.049491 & -10.8766 & -19.5374 & 0.183244 & 0.049741 \\
\hline Dec-93 & 0.270011 & 0.020251 & 0.075002 & -59.5463 & -1.95138 & 0.228418 & 0.08866 \\
\hline Mar-94 & 0.317887 & 0.017952 & 0.056473 & -132.943 & -42.2092 & 0.302313 & 0.059382 \\
\hline Jun-94 & 0.288806 & 0.016683 & 0.057766 & -114.251 & -45.1711 & 0.284484 & 0.058644 \\
\hline Sep-94 & 0.272844 & 0.017734 & 0.064998 & -106.525 & & 0.272937 & 0.064976 \\
\hline Dec-94 & 0.247082 & 0.015941 & 0.064516 & -57.7143 & -22.5979 & 0.247069 & 0.064519 \\
\hline Mar-95 & 0.199133 & 0.015073 & 0.075695 & -31.5354 & -8.63664 & 0.212415 & 0.070961 \\
\hline Jun-95 & 0.175437 & 0.016823 & 0.09589 & -17.0564 & -2.5103 & 0.178284 & 0.094358 \\
\hline Sep-95 & 0.17913 & 0.013775 & 0.076898 & -30.4279 & -5.90612 & 0.184507 & 0.074657 \\
\hline Dec-95 & 0.177157 & 0.014263 & 0.080508 & -25.0082 & -3.0539 & 0.170113 & 0.083842 \\
\hline Mar-96 & 0.18226 & 0.033669 & 0.184729 & -13.9286 & -4.22365 & 0.190191 & 0.177026 \\
\hline Jun-96 & 0.189256 & 0.031383 & 0.165821 & -8.14555 & -3.582 & 0.186883 & 0.167927 \\
\hline Sep-96 & 0.214443 & 0.019293 & 0.089969 & -25.3476 & -20.7501 & 0.194036 & 0.099431 \\
\hline Dec-96 & 0.206392 & 0.027553 & 0.133499 & -20.9685 & -17.2644 & 0.213744 & 0.128908 \\
\hline Mar-97 & 0.225355 & 0.033153 & 0.147113 & -4.97983 & -4.04541 & 0.226508 & 0.146365 \\
\hline Jun-97 & 0.210704 & 0.030587 & 0.145167 & -5.81933 & -9.29391 & 0.211154 & 0.144858 \\
\hline Sep-97 & 0.226944 & 0.07965 & 0.350965 & -3.37598 & -2.0774 & 0.228543 & 0.348511 \\
\hline Dec-97 & 0.205724 & 0.064373 & 0.312911 & -4.01892 & -5.36846 & 0.212895 & 0.302371 \\
\hline Mar-98 & 0.179181 & 0.041799 & 0.233278 & -8.99493 & -8.22499 & 0.185899 & 0.224848 \\
\hline Jun-98 & 0.104539 & 0.035679 & 0.341302 & -34.7595 & -15.1658 & 0.13261 & 0.269054 \\
\hline Sep-98 & 0.123777 & 0.046525 & 0.375879 & -37.8915 & -21.006 & 0.113344 & 0.410477 \\
\hline Dec-98 & 0.102027 & 0.046324 & 0.454033 & -30.9073 & -19.1454 & 0.103997 & 0.445432 \\
\hline Mar-99 & 0.108744 & 0.047943 & 0.440879 & -20.8898 & -11.1101 & 0.10067 & 0.476242 \\
\hline Jun-99 & 0.105703 & 0.08752 & 0.827979 & -12.4448 & -4.22247 & 0.111267 & 0.786573 \\
\hline Sep-99 & 0.116049 & 0.134402 & 1.158145 & -11.5551 & -0.81483 & 0.117029 & 1.148445 \\
\hline Dec-99 & 0.127868 & 0.121039 & 0.946597 & -5.95818 & -4.73502 & 0.119755 & 1.010724 \\
\hline Mar-00 & 0.174397 & 0.230984 & 1.324475 & -45.1537 & -25.9315 & 0.166217 & 1.389654 \\
\hline Jun-00 & 0.134107 & 0.172152 & 1.283688 & -15.0463 & -21.1222 & 0.144599 & 1.190546 \\
\hline Sep-0O & 0.136289 & 0.104168 & 0.764318 & -17.5327 & -17.3031 & 0.134514 & 0.774404 \\
\hline Dec-0O & 0.124765 & 0.093861 & 0.752305 & -3.65767 & -6.43875 & 0.12101 & 0.775652 \\
\hline Mar-01 & 0.107163 & 0.094513 & 0.881964 & -17.7588 & -1.49316 & 0.114089 & 0.828419 \\
\hline Jun-01 & 0.108098 & 0.061437 & 0.568345 & -9.23343 & -5.68007 & 0.108578 & 0.565827 \\
\hline
\end{tabular}


* Given the nature of the data of P. Errors, no average indicators can be constructed for it.

We repeat the entire process with the share prices of Pakistan's stock market and US stock prices to arrive at another indicator of ICAPM pricing errors (see Table 1). Unfortunately, the KSE 100 Index could not be incorporated to obtain pricing errors due to data constraints.

\section{Indexes of Stock Market Development}

In order to obtain a measure of overall stock market development, aggregate indexes of the indicators discussed above are constructed. Three indexes may be constructed:

\section{Index 1}

The stock market size and liquidity are two such sectors, which largely determine the level of overall development of the stock market. Therefore an aggregate index of means removed ratios of market capitalisation, value traded to GDP and turnover is constructed to look into the overall level of development (Table 2).

\section{Index 2}

In Index 2, we add ICAPM pricing error as obtained from the regression of SBP Index on S\&P 500 Index as a measure of Market Integration to Index 1(Table 2).

\section{Index 3}

Index 3 is similar to Index 2 except that in place of ICAPM pricing errors obtained from the regression of SBP Index on S\&P 500 index, we introduce ICAPM pricing errors obtained from the regression of Pakistan's stock prices on US stock prices as a measure of market integration (Table 2).

\section{Indicators to Gauge Financial Intermediary Development}

The level of financial intermediary development is depicted theoretically in the form of development in different sectors of financial intermediation namely, scheduled banking sector, development finance institutions, national savings schemes etc. The following indicators are constructed in this paper. 


\section{Size of the Financial System}

In order to measure the size of the financial system, the ratio of the liquid liabilities of the financial intermediaries to GDP is obtained which is defined as $\mathrm{M}_{2}$ money supply divided by GDP (Table $3 \mathrm{~A}$ and $3 \mathrm{~B}$ ). $\mathrm{M}_{2}$ money supply comprises currency credits, demand deposits with scheduled banks, other deposits with the State Bank of Pakistan and time deposits. If the size of the financial system is positively related to the provision of financial services, then this ratio is a good indicator of the provision of financial intermediary services.

The ratio of quasi-liquid liabilities to GDP is constructed as another indicator of the size of the financial system. It is defined as $M_{2}$ minus $M_{1}$ money supply divided by GDP (Table $3 \mathrm{~A}$ and $3 \mathrm{~B}$ ). $\mathrm{M}_{1}$ only includes currency credits, demand deposits with scheduled banks and other deposits with the SBP. This indicator subtracts narrow money from liquid liabilities. Sometimes a quasi-liquid measure is preferred instead of liquid liabilities because $M_{1}$ represents highly liquid bank deposits and therefore may not be as closely associated with efficient financial intermediation as longer term investments by financial intermediaries. The quasi-liquid measure focuses on longer-term liabilities. 
Atif Naveed 163

Table 2: Indexes of Stock Market Development

\begin{tabular}{|c|c|c|c|c|c|c|}
\hline Year & Index 1 & $\begin{array}{l}\text { Index 1 } \\
\text { (Av.Values) }\end{array}$ & Index 2 & $\begin{array}{l}\text { Index } 2 \\
\text { (Av.Values) }\end{array}$ & Index 3 & $\begin{array}{l}\text { Index } 3 \\
\text { (Av.Values) }\end{array}$ \\
\hline Sep-90 & -0.8083 & -0.80783 & -1.12607 & -1.12571 & -0.9901 & -0.98974 \\
\hline Dec-90 & -0.81079 & -0.80908 & -1.08842 & -1.08715 & -1.0838 & -1.08253 \\
\hline Mar-91 & -0.79557 & -0.79928 & -1.02065 & -1.02344 & -0.41982 & -0.42261 \\
\hline Jun-91 & -0.75229 & -0.75643 & -0.85209 & -0.8552 & -0.40918 & -0.41228 \\
\hline Sep-91 & -0.69093 & -0.691 & -0.59486 & -0.59491 & -0.85078 & -0.85083 \\
\hline Dec-91 & -0.54777 & -0.60287 & -0.26209 & -0.30342 & -0.52439 & -0.56572 \\
\hline Mar-92 & -0.56452 & -0.55212 & -0.20324 & -0.19394 & -0.37489 & -0.36558 \\
\hline Jun-92 & -0.54557 & -0.5508 & -0.23824 & -0.24217 & -0.29881 & -0.30274 \\
\hline Sep-92 & -0.56342 & -0.56555 & -0.29894 & -0.30054 & -0.52842 & -0.53003 \\
\hline Dec-92 & -0.5608 & -0.56282 & -0.26325 & -0.26476 & -0.51583 & -0.51734 \\
\hline Mar-93 & -0.59957 & -0.58305 & -0.37466 & -0.36227 & -0.56008 & -0.54769 \\
\hline Jun-93 & -0.57452 & -0.5723 & -0.28742 & -0.28576 & -0.58894 & -0.58728 \\
\hline Sep-93 & -0.50704 & -0.50514 & -0.21379 & -0.21238 & -0.43913 & -0.43771 \\
\hline Dec-93 & -0.21989 & -0.28238 & -0.37215 & -0.41902 & 0.054238 & 0.007366 \\
\hline Mar-94 & -0.16429 & -0.18626 & -0.89405 & -0.91052 & -0.54046 & -0.55694 \\
\hline Jun-94 & -0.23047 & -0.23277 & -0.80015 & -0.80187 & -0.63692 & -0.63864 \\
\hline Sep-94 & -0.24556 & -0.24031 & -0.75214 & -0.7482 & -0.63357 & -0.62964 \\
\hline Dec-94 & -0.31134 & -0.30673 & -0.42667 & -0.42322 & -0.34073 & -0.33727 \\
\hline Mar-95 & -0.4005 & -0.37552 & -0.29253 & -0.27379 & -0.18691 & -0.16816 \\
\hline Jun-95 & -0.4107 & -0.40325 & -0.18899 & -0.18341 & -0.0977 & -0.09212 \\
\hline Sep-95 & -0.44904 & -0.43736 & -0.32043 & -0.31167 & -0.18015 & -0.17139 \\
\hline Dec-95 & -0.445 & -0.45182 & -0.27578 & -0.28089 & -0.13202 & -0.13714 \\
\hline Mar-96 & -0.16306 & -0.15197 & 0.020752 & 0.029071 & 0.060938 & 0.069256 \\
\hline Jun-96 & -0.18874 & -0.18704 & 0.045901 & 0.047172 & 0.051824 & 0.053094 \\
\hline Sep-96 & -0.32057 & -0.34643 & -0.18507 & -0.20446 & -0.31845 & -0.33784 \\
\hline Dec-96 & -0.22175 & -0.20808 & -0.07732 & -0.06707 & -0.18923 & -0.17897 \\
\hline Mar-97 & -0.1244 & -0.11835 & 0.118463 & 0.123001 & 0.092752 & 0.09729 \\
\hline Jun-97 & -0.17595 & -0.17107 & 0.073351 & 0.077012 & -0.02888 & -0.02522 \\
\hline Sep-97 & 0.478148 & 0.484048 & 0.582688 & 0.587113 & 0.575772 & 0.580197 \\
\hline Dec-97 & 0.271939 & 0.279249 & 0.423094 & 0.428577 & 0.369089 & 0.374572 \\
\hline Mar-98 & -0.04974 & -0.04175 & 0.143626 & 0.149622 & 0.082674 & 0.08867 \\
\hline Jun-98 & -0.12379 & -0.1465 & -0.10975 & -0.12678 & -0.08258 & -0.09961 \\
\hline Sep-98 & 0.039438 & 0.061689 & -0.01138 & 0.005311 & -0.05248 & -0.0358 \\
\hline Dec-98 & 0.083239 & 0.081136 & 0.075103 & 0.073525 & 0.009778 & 0.008201 \\
\hline Mar-99 & 0.094491 & 0.122335 & 0.160462 & 0.181345 & 0.14524 & 0.166123 \\
\hline Jun-99 & 0.840426 & 0.809888 & 0.78476 & 0.761857 & 0.813571 & 0.790667 \\
\hline Sep-99 & 1.606344 & 1.604262 & 1.36603 & 1.364468 & 1.441878 & 1.440315 \\
\hline Dec-99 & 1.284344 & 1.347285 & 1.167507 & 1.214713 & 1.138407 & 1.185612 \\
\hline Mar-OO & 2.675913 & 2.742346 & 1.910215 & 1.960039 & 1.84701 & 1.896834 \\
\hline Jun-00 & 2.083514 & 2.00648 & 1.6971 & 1.639324 & 1.478735 & 1.42096 \\
\hline Sep-00 & 0.960929 & 0.974502 & 0.836069 & 0.846248 & 0.69717 & 0.707349 \\
\hline Dec-00 & 0.842794 & 0.867224 & 0.85401 & 0.872332 & 0.780312 & 0.798634 \\
\hline Mar-01 & 0.959874 & 0.918521 & 0.833542 & 0.802527 & 0.946302 & 0.915286 \\
\hline Jun-01 & 0.344478 & 0.346905 & 0.437458 & 0.439279 & 0.418568 & 0.420388 \\
\hline
\end{tabular}


In the case of Pakistan, in view of the significant role of the public sector in economic activity, it would be desirable to include credit extended to the public sector as well apart from the private sector. Therefore, total credit extended divided by GDP is also included as another indicator (Table $3 \mathrm{~A}$ and 3B).

\section{Scheduled Banks}

In order to measure the level of development of the banking system, we use the ratio of the total claims of scheduled banks to GDP, which in other words is the ratio of domestic assets of scheduled banks to GDP (Table $3 \mathrm{~A}$ and 3B). This indicator shows the size of the banking system.

\section{Non - Bank Financial Corporations}

So as to observe the level of development of the non-bank financial system, the ratio of assets of non-bank financial intermediaries to GDP is calculated (Table $3 \mathrm{~A}$ and $3 \mathrm{~B}$ ). It indicates the size of the non-bank financial corporations (NBFCs). The non-bank financial corporations include Development Finance Institutions, Investment banks, Leasing Companies, Modarbas, Discount Houses, Housing Finance Companies and Venture Capital Companies.

\section{National Saving Schemes}

National Saving Schemes have been a prominent player in the financial sector of Pakistan. The outstanding amount of the national saving schemes divided by GDP is taken as an indicator to measure the role played by these schemes in mobilising savings and hence to determine their level of development (Table $3 \mathrm{~A}$ and $3 \mathrm{~B}$ ). The schemes include prize bonds ranging from Rs. 5 to Rs. 1000, certificates such as Defence Saving Certificates, "Khas" Deposit Certificates, Premium Saving Certificates, National Deposit Certificates and accounts such as "Mahana Amdani" Accounts, Special Saving Accounts, National Deposit Accounts and Saving Accounts.

\section{Indexes of Financial Intermediary Development}

In order to produce a measure of the overall financial intermediary development, the aggregate indexes of the above-discussed indicators are constructed. In this regard, three aggregate indexes are constructed. These financial indexes are written in brief as FINDEXES.

\section{Findex 1}

Financial index 1 averages the means removed values of the ratio of liquid liabilities to GDP and the ratio of total domestic credit to GDP (Table 4). Liquid liabilities indicate the financial depth from the liability side while the total domestic credit represents the asset side of the financial 
Atif Naveed 165

depth i.e. of the received deposits, which are in fact liabilities, how much is channelised properly into developmental investment. 
Table 3A: Indicators of Financial Intermediary Development

\begin{tabular}{|c|c|c|c|c|c|c|c|}
\hline Year & M2/GDP & $\begin{array}{l}\text { Credit to } \\
\text { Pvt. } \\
\text { Sector/ } \\
\text { GDP }\end{array}$ & $\begin{array}{l}\text { Total } \\
\text { credit } \\
\text { extended/ } \\
\text { GDP }\end{array}$ & $\begin{array}{l}\text { M2-M1/ } \\
\text { GDP }\end{array}$ & $\begin{array}{l}\text { Assets of } \\
\text { Sch. } \\
\text { Banks / } \\
\text { GDP }\end{array}$ & $\begin{array}{l}\text { Assets of } \\
\text { NBFIs/ } \\
\text { GDP }\end{array}$ & $\begin{array}{l}\text { NSS } \\
\text { Amounts/ } \\
\text { GDP }\end{array}$ \\
\hline Sep-90 & 0.440592 & 0.217799 & 0.402913 & 0.135927 & 0.835583 & 0.180588 & 0.198473 \\
\hline Dec-90 & 0.452853 & 0.219622 & 0.391838 & 0.133361 & 0.843476 & 0.182374 & 0.188666 \\
\hline Mar-91 & 0.44598 & 0.214979 & 0.376278 & 0.128811 & 0.820609 & 0.185268 & 0.185872 \\
\hline Jun-91 & 0.442946 & 0.214305 & 0.398591 & 0.14981 & 0.853896 & 0.185517 & 0.177366 \\
\hline Sep-91 & 0.434644 & 0.202719 & 0.398899 & 0.131541 & 0.8457 & 0.196088 & 0.172281 \\
\hline Dec-91 & 0.444002 & 0.209292 & 0.395574 & 0.158626 & 0.878021 & 0.203573 & 0.165312 \\
\hline Mar-92 & 0.460251 & 0.212235 & 0.408031 & 0.143489 & 0.844046 & 0.211698 & 0.158496 \\
\hline Jun-92 & 0.471718 & 0.207373 & 0.414577 & 0.189091 & 0.873885 & 0.216373 & 0.152776 \\
\hline Sep-92 & 0.487849 & 0.218311 & 0.441106 & 0.209568 & 0.90453 & 0.220223 & 0.150237 \\
\hline Dec-92 & 0.517731 & 0.230044 & 0.455906 & 0.225059 & 0.952741 & 0.222337 & 0.145504 \\
\hline Mar-93 & 0.507109 & 0.232079 & 0.443955 & 0.218933 & 0.939367 & 0.225301 & 0.143816 \\
\hline Jun-93 & 0.499682 & 0.232096 & 0.451326 & 0.224557 & 0.961948 & 0.226262 & 0.144086 \\
\hline Sep-93 & 0.487725 & 0.222265 & 0.452129 & 0.229914 & 0.961739 & 0.232068 & 0.142942 \\
\hline Dec-93 & 0.503996 & 0.233837 & 0.418334 & 0.236995 & 0.973644 & 0.234785 & 0.150528 \\
\hline Mar-94 & 0.504055 & 0.229515 & 0.438133 & 0.2419 & 0.96094 & 0.238708 & 0.147437 \\
\hline Jun-94 & 0.502118 & 0.225877 & 0.447046 & 0.246013 & 0.997848 & 0.239352 & 0.14884 \\
\hline Sep-94 & 0.485823 & 0.21904 & 0.433262 & 0.240365 & 0.985146 & 0.23495 & 0.150441 \\
\hline Dec-94 & 0.50543 & 0.229257 & 0.442847 & 0.244285 & 1.02302 & 0.227678 & 0.14236 \\
\hline Mar-95 & 0.49017 & 0.228089 & 0.419724 & 0.234184 & 0.982476 & 0.222352 & 0.152108 \\
\hline Jun-95 & 0.493269 & 0.224888 & 0.430894 & 0.240192 & 0.989304 & 0.215374 & 0.152827 \\
\hline Sep-95 & 0.475649 & 0.215704 & 0.422219 & 0.237957 & 0.973164 & 0.211338 & 0.149646 \\
\hline Dec-95 & 0.482742 & 0.235186 & 0.426451 & 0.238149 & 0.958188 & 0.20552 & 0.149208 \\
\hline Mar-96 & 0.484237 & 0.231157 & 0.414546 & 0.240308 & 0.949336 & 0.201234 & 0.161035 \\
\hline Jun-96 & 0.48639 & 0.225937 & 0.423578 & 0.254248 & 0.989896 & 0.1954 & 0.157467 \\
\hline Sep-96 & 0.466298 & 0.219905 & 0.432866 & 0.253539 & 0.957314 & 0.193949 & 0.157176 \\
\hline Dec-96 & 0.472493 & 0.235534 & 0.443261 & 0.258356 & 1.028182 & 0.190564 & 0.155961 \\
\hline Mar-97 & 0.474898 & 0.23082 & 0.42213 & 0.270826 & 0.945243 & 0.188128 & 0.16243 \\
\hline Jun-97 & 0.473028 & 0.226961 & 0.43429 & 0.273821 & 0.956213 & 0.184274 & 0.16722 \\
\hline Sep-97 & 0.466223 & 0.218192 & 0.432999 & 0.28026 & 0.918179 & 0.175659 & 0.175556 \\
\hline Dec-97 & 0.499021 & 0.237217 & 0.441203 & 0.273742 & 0.942324 & 0.166181 & 0.182489 \\
\hline Mar-98 & 0.499404 & 0.243 & 0.442649 & 0.31035 & 0.923156 & 0.157924 & 0.18676 \\
\hline Jun-98 & 0.486246 & 0.238332 & 0.447115 & 0.292634 & 0.63993 & 0.148858 & 0.184993 \\
\hline Sep-98 & 0.476979 & 0.223612 & 0.429725 & 0.262635 & 0.629922 & 0.144499 & 0.189635 \\
\hline Dec-98 & 0.484938 & 0.24727 & 0.430404 & 0.246312 & 0.62317 & 0.139372 & 0.190192 \\
\hline Mar-99 & 0.469044 & 0.246309 & 0.416148 & 0.23689 & 0.597227 & 0.135118 & 0.19637 \\
\hline Jun-99 & 0.468046 & 0.246843 & 0.431476 & 0.23301 & 0.633898 & 0.130266 & 0.202633 \\
\hline Sep-99 & 0.45615 & 0.237753 & 0.414046 & 0.223171 & 0.626284 & 0.128025 & 0.236628 \\
\hline Dec-99 & 0.466105 & 0.248487 & 0.403233 & 0.206076 & 0.629264 & 0.125304 & 0.232576 \\
\hline Mar-00 & 0.460698 & 0.247282 & 0.3884 & 0.205975 & 0.594339 & 0.123062 & 0.242543 \\
\hline Jun-00 & 0.479342 & 0.237274 & 0.396222 & 0.226421 & 0.618268 & 0.120363 & 0.244611 \\
\hline Sep-00 & 0.470195 & 0.233386 & 0.394682 & 0.237859 & 0.618903 & 0.118028 & 0.242701 \\
\hline Dec-00 & 0.486004 & 0.255548 & 0.414848 & 0.227507 & 0.625695 & 0.11511 & 0.239575 \\
\hline $\begin{array}{l}\text { Mar-01 } \\
\text { Jun-01 }\end{array}$ & $\begin{array}{l}0.47431 \\
0.482826\end{array}$ & $\begin{array}{l}0.251063 \\
0.236936\end{array}$ & $\begin{array}{l}0.39531 \\
0.391827\end{array}$ & $\begin{array}{l}0.234506 \\
0.241886\end{array}$ & $\begin{array}{l}0.602941 \\
0.627443\end{array}$ & $\begin{array}{l}0.112784 \\
0.109996\end{array}$ & $\begin{array}{l}0.24005 \\
0.240899\end{array}$ \\
\hline
\end{tabular}


Table 3B: Indicators of Financial Intermediary Development

\begin{tabular}{|c|c|c|c|c|c|c|c|}
\hline Year & $\begin{array}{l}\text { M2/GDP } \\
\text { (AV. } \\
\text { Values) }\end{array}$ & $\begin{array}{l}\text { Credit to } \\
\text { Pvt. } \\
\text { Sector/ } \\
\text { GDP(AV. } \\
\text { Values) }\end{array}$ & $\begin{array}{l}\text { Credit } \\
\text { extended/ } \\
\text { GDP } \\
\text { (AV) }\end{array}$ & $\begin{array}{l}\text { M2-M1/ } \\
\text { GDP(AV. } \\
\text { Values) }\end{array}$ & $\begin{array}{l}\text { Assets of } \\
\text { Sch. } \\
\text { Banks/ } \\
\text { GDP(AV. } \\
\text { Values) }\end{array}$ & $\begin{array}{l}\text { Assets of } \\
\text { NBFIs/GD } \\
\text { P(AV. } \\
\text { Value) }\end{array}$ & $\begin{array}{l}\text { NSS } \\
\text { Amount/ } \\
\text { GDP(AV. } \\
\text { Values) }\end{array}$ \\
\hline Sep-90 & 0.436796 & 0.21505 & 0.396297 & 0.135927 & 0.824193 & 0.177004 & 0.198127 \\
\hline Dec-90 & 0.435898 & 0.215753 & 0.387179 & 0.13107 & 0.817192 & 0.178959 & 0.188977 \\
\hline Mar-91 & 0.44294 & 0.212946 & 0.377373 & 0.129999 & 0.813688 & 0.181983 & 0.183305 \\
\hline Jun-91 & 0.432945 & 0.206694 & 0.37677 & 0.132519 & 0.819782 & 0.182394 & 0.177173 \\
\hline Sep-91 & 0.432198 & 0.201568 & 0.392262 & 0.130549 & 0.836589 & 0.19039 & 0.172092 \\
\hline Dec-91 & 0.435161 & 0.204319 & 0.384985 & 0.140618 & 0.845449 & 0.198132 & 0.164895 \\
\hline Mar-92 & 0.449493 & 0.208514 & 0.407764 & 0.135411 & 0.838489 & 0.20646 & 0.158106 \\
\hline Jun-92 & 0.455623 & 0.206036 & 0.403605 & 0.157595 & 0.848969 & 0.211389 & 0.152242 \\
\hline Sep-92 & 0.476898 & 0.211217 & 0.429152 & 0.201048 & 0.894001 & 0.217356 & 0.150332 \\
\hline Dec-92 & 0.489633 & 0.221785 & 0.443561 & 0.209436 & 0.912146 & 0.219551 & 0.145991 \\
\hline Mar-93 & 0.499429 & 0.22782 & 0.446312 & 0.205968 & 0.928709 & 0.22258 & 0.142857 \\
\hline Jun-93 & 0.492133 & 0.227801 & 0.440937 & 0.219455 & 0.932259 & 0.223625 & 0.142418 \\
\hline Sep-93 & 0.486222 & 0.221782 & 0.448141 & 0.226695 & 0.952206 & 0.227625 & 0.142133 \\
\hline Dec-93 & 0.486249 & 0.22391 & 0.431273 & 0.230291 & 0.947649 & 0.230535 & 0.142699 \\
\hline Mar-94 & 0.49648 & 0.226619 & 0.431398 & 0.236201 & 0.94323 & 0.23461 & 0.144673 \\
\hline Jun-94 & 0.491862 & 0.219002 & 0.438084 & 0.237498 & 0.961765 & 0.235444 & 0.144928 \\
\hline Sep-94 & 0.482677 & 0.217954 & 0.431612 & 0.237458 & 0.971582 & 0.233528 & 0.14849 \\
\hline Dec-94 & 0.483888 & 0.219345 & 0.434033 & 0.23604 & 0.976286 & 0.226325 & 0.143304 \\
\hline Mar-95 & 0.486013 & 0.226768 & 0.423236 & 0.22921 & 0.965582 & 0.221053 & 0.142891 \\
\hline Jun-95 & 0.478233 & 0.219507 & 0.417514 & 0.231788 & 0.956007 & 0.214138 & 0.145938 \\
\hline Sep-95 & 0.475947 & 0.215727 & 0.421744 & 0.237415 & 0.963142 & 0.210516 & 0.147955 \\
\hline Dec-95 & 0.470751 & 0.222842 & 0.412602 & 0.235024 & 0.94631 & 0.20473 & 0.146969 \\
\hline Mar-96 & 0.481828 & 0.230845 & 0.415117 & 0.231329 & 0.934396 & 0.20047 & 0.1555 \\
\hline Jun-96 & 0.477256 & 0.222989 & 0.414145 & 0.245255 & 0.950561 & 0.194666 & 0.155497 \\
\hline Sep-96 & 0.470524 & 0.217204 & 0.434886 & 0.254481 & 0.956848 & 0.192557 & 0.156472 \\
\hline Dec-96 & 0.464968 & 0.229461 & 0.431497 & 0.25412 & 0.975867 & 0.189225 & 0.154103 \\
\hline Mar-97 & 0.470249 & 0.23122 & 0.41918 & 0.263546 & 0.947106 & 0.186834 & 0.158229 \\
\hline Jun-97 & 0.465074 & 0.222545 & 0.419897 & 0.268008 & 0.934129 & 0.183031 & 0.162731 \\
\hline Sep-97 & 0.463825 & 0.21808 & 0.429488 & 0.276718 & 0.912492 & 0.177159 & 0.173802 \\
\hline Dec-97 & 0.478057 & 0.224963 & 0.432696 & 0.281267 & 0.919787 & 0.167637 & 0.178445 \\
\hline Mar-98 & 0.498307 & 0.240742 & 0.439511 & 0.30267 & 0.914812 & 0.159345 & 0.185387 \\
\hline Jun-98 & 0.484302 & 0.236379 & 0.440293 & 0.298196 & 0.814514 & 0.150235 & 0.183608 \\
\hline Sep-98 & 0.474076 & 0.223654 & 0.424794 & 0.266624 & 0.623426 & 0.144923 & 0.189664 \\
\hline Dec-98 & 0.473605 & 0.236087 & 0.42269 & 0.251245 & 0.617134 & 0.139785 & 0.189507 \\
\hline Mar-99 & 0.467175 & 0.24475 & 0.413453 & 0.239639 & 0.590468 & 0.135521 & 0.193007 \\
\hline Jun-99 & 0.456985 & 0.241967 & 0.415992 & 0.22948 & 0.609041 & 0.130659 & 0.198674 \\
\hline Sep-99 & 0.455743 & 0.237975 & 0.410961 & 0.223739 & 0.620157 & 0.128166 & 0.232466 \\
\hline Dec-99 & 0.456465 & 0.241301 & 0.401344 & 0.209692 & 0.618035 & 0.125443 & 0.231628 \\
\hline Mar-00 & 0.460073 & 0.246976 & 0.391988 & 0.205753 & 0.591058 & 0.123199 & 0.237618 \\
\hline Jun-00 & 0.466231 & 0.237894 & 0.390982 & 0.222657 & 0.604011 & 0.120497 & 0.242254 \\
\hline Sep-0O & 0.467235 & 0.23189 & 0.39022 & 0.231502 & 0.608993 & 0.118138 & 0.241983 \\
\hline Dec-00 & 0.472364 & 0.244456 & 0.392779 & 0.221019 & 0.614149 & 0.115217 & 0.235518 \\
\hline Mar-01 & 0.472352 & 0.251186 & 0.387032 & 0.231059 & 0.599127 & 0.112889 & 0.238613 \\
\hline Jun-01 & 0.469631 & 0.239999 & 0.384593 & 0.234681 & 0.603478 & 0.110099 & 0.238198 \\
\hline
\end{tabular}


Table 4: Indexes of Financial Intermediary Development

\begin{tabular}{|c|c|c|c|c|c|c|}
\hline Year & Findex 1 & $\begin{array}{l}\text { Findex } 1 \\
\text { (av.values) }\end{array}$ & Findex 2 & $\begin{array}{l}\text { Findex } 2 \\
\text { (av.values) }\end{array}$ & Findex 3 & $\begin{array}{l}\text { Findex } 3 \\
\text { (av.values) }\end{array}$ \\
\hline Sep-90 & -0.06103 & 0.031482 & -0.00507 & -0.058 & 0.032472 & -0.00334 \\
\hline Dec-90 & -0.06133 & 0.019571 & -0.0165 & -0.06993 & 0.015964 & -0.01957 \\
\hline Mar-91 & -0.08697 & 0.010591 & -0.02927 & -0.07423 & 0.009409 & -0.02558 \\
\hline Jun-91 & -0.06369 & 0.008355 & -0.02919 & -0.0856 & 0.001029 & -0.03939 \\
\hline Sep-91 & -0.07201 & 0.014946 & -0.02597 & -0.06775 & 0.012932 & -0.02661 \\
\hline Dec-91 & -0.06616 & 0.028425 & -0.02254 & -0.07335 & 0.017167 & -0.02891 \\
\hline Mar-92 & -0.03438 & 0.017104 & -0.00505 & -0.03068 & 0.016891 & -0.00568 \\
\hline Jun-92 & -0.01462 & 0.026792 & 0.003238 & -0.02916 & 0.019111 & -0.00642 \\
\hline Sep-92 & 0.03373 & 0.041231 & 0.029138 & 0.024239 & 0.044624 & 0.025829 \\
\hline Dec-92 & 0.082557 & 0.055353 & 0.049825 & 0.055139 & 0.047764 & 0.038158 \\
\hline Mar-93 & 0.057266 & 0.052326 & 0.038881 & 0.06888 & 0.054091 & 0.044776 \\
\hline Jun-93 & 0.058234 & 0.063529 & 0.04106 & 0.054643 & 0.056615 & 0.03848 \\
\hline Sep-93 & 0.046672 & 0.071927 & 0.04164 & 0.05702 & 0.071479 & 0.044797 \\
\hline Dec-93 & 0.023624 & 0.095767 & 0.04446 & 0.036748 & 0.076079 & 0.039487 \\
\hline Mar-94 & 0.047165 & 0.09214 & 0.057283 & 0.04779 & 0.085547 & 0.053442 \\
\hline Jun-94 & 0.055708 & 0.110552 & 0.064402 & 0.05092 & 0.095024 & 0.056522 \\
\hline Sep-94 & 0.022307 & 0.10046 & 0.043905 & 0.033352 & 0.102173 & 0.050137 \\
\hline Dec-94 & 0.054196 & 0.087083 & 0.038568 & 0.037555 & 0.080983 & 0.034927 \\
\hline Mar-95 & 0.010801 & 0.079481 & 0.023211 & 0.026823 & 0.066175 & 0.021685 \\
\hline Jun-95 & 0.027291 & 0.07077 & 0.022895 & 0.011653 & 0.055329 & 0.008855 \\
\hline Sep-95 & -0.00144 & 0.051062 & -0.00146 & 0.014311 & 0.055336 & 0.008036 \\
\hline Dec-95 & 0.011005 & 0.033676 & -0.00383 & -0.00222 & 0.03603 & -0.00963 \\
\hline Mar-96 & -0.00155 & 0.044407 & 0.000573 & 0.012596 & 0.039506 & 0.00398 \\
\hline Jun-96 & 0.011415 & 0.043142 & -0.00594 & 0.006559 & 0.035302 & -0.00707 \\
\hline Sep-96 & 0.0014 & 0.027044 & -0.01334 & 0.024354 & 0.035786 & 0.000294 \\
\hline Dec-96 & 0.020213 & 0.046653 & -0.01028 & 0.014359 & 0.032816 & -0.01267 \\
\hline Mar-97 & -0.00233 & 0.021429 & -0.01584 & 0.005158 & 0.024634 & -0.01473 \\
\hline Jun-97 & 0.010133 & 0.027665 & -0.00818 & 0.000511 & 0.020911 & -0.01593 \\
\hline Sep-97 & 0.00148 & 0.012409 & -0.01266 & 0.010726 & 0.022305 & -0.00325 \\
\hline Dec-97 & 0.045537 & 0.017581 & 0.006079 & 0.029738 & 0.016457 & -0.00033 \\
\hline Mar-98 & 0.047654 & 0.002863 & 0.001793 & 0.059499 & 0.012288 & 0.012927 \\
\hline Jun-98 & 0.039178 & -0.12917 & -0.01735 & 0.04553 & -0.04823 & -0.00918 \\
\hline Sep-98 & 0.008855 & -0.13244 & -0.03199 & 0.015989 & -0.12345 & -0.02271 \\
\hline Dec-98 & 0.017991 & -0.14345 & -0.03367 & 0.012956 & -0.13576 & -0.03156 \\
\hline Mar-99 & -0.01555 & -0.14997 & -0.04763 & -0.00501 & -0.14773 & -0.04147 \\
\hline Jun-99 & 0.001582 & -0.13263 & -0.03695 & -0.0128 & -0.13851 & -0.04406 \\
\hline Sep-99 & -0.03154 & -0.0763 & -0.009 & -0.02018 & -0.07476 & -0.00329 \\
\hline Dec-99 & -0.03394 & -0.08765 & -0.01961 & -0.03098 & -0.08222 & -0.01365 \\
\hline Mar-00 & -0.05719 & -0.08698 & -0.02035 & -0.0384 & -0.08589 & -0.01197 \\
\hline Jun-00 & -0.0284 & -0.07858 & -0.00677 & -0.03306 & -0.0769 & -0.00646 \\
\hline Sep-OO & -0.0398 & -0.08616 & -0.01834 & -0.03291 & -0.07976 & -0.01003 \\
\hline Dec-00 & 0.000658 & -0.09464 & -0.00649 & -0.02436 & -0.09529 & -0.01897 \\
\hline Mar-01 & -0.03475 & -0.10702 & -0.02672 & -0.03129 & -0.09977 & -0.02126 \\
\hline Jun-01 & -0.02997 & -0.10083 & -0.02696 & -0.03713 & -0.10395 & -0.02862 \\
\hline
\end{tabular}

Findex 2

This combines the means removed values of the ratio of total assets of scheduled banks to GDP, the ratio of assets of non-bank financial 
corporations to GDP, and the amounts of saving schemes to GDP (Table 4). This aggregate financial index averages the information on all the sectors of financial intermediation from the asset side.

\section{Findex 3}

This averages information on the ratio of liquid liabilities to GDP, the ratio of total domestic credit to GDP, the ratio of assets of private nonbank financial corporations to GDP, and outstanding amounts of national saving schemes to GDP. This index measures development from a slightly different angle (Table 4).

\section{Do Stock Market Development and Financial Intermediary Development Go Together in Pakistan?}

It has been revealed through cross-country analysis that a strong positive linkage exists between stock market development and financial intermediary development. In order to ascertain whether stock market development goes hand in hand with the development of the bank and nonbank sector in Pakistan, research has been carried out in this paper. Moreover, an attempt has also been made to see if there is any strong association within the two sectors amongst their respective indicators. This will help in ascertaining how integrated development has been within each of the two sectors over the period of study. The statistical approach followed in this regard is the correlation analysis. The following queries have been probed:

1. Degree of linkage between various indicators of stock market development.

2. The extent of relationship between indicators of financial intermediary development.

3. The degree of relationship between the indexes of stock market development.

4. The extent of linkage between the aggregate indexes of the financial sector's development.

5. The linkage between individual indicators of the stock market and the financial sector's development.

6. The relationship between aggregate indexes of the two sectors. 


\section{Linkage Between Various Indicators of Stock Market Development}

Table 5: Correlation Among Indicators of Stock Market Development

\begin{tabular}{|c|c|c|c|c|c|c|}
\hline & Variables & 1 & 2 & 3 & 4 & 5 \\
\hline 1 & $\begin{array}{l}\text { Market Cap to } \\
\text { GDP }\end{array}$ & 1.00 & $\begin{array}{l}-0.200 \\
(0.193)\end{array}$ & $\begin{array}{l}-0.355^{*} \\
(0.018)\end{array}$ & $\begin{array}{l}-0.195 \\
(0.204)\end{array}$ & $\begin{array}{l}-0.177 \\
(0.250)\end{array}$ \\
\hline 2. & $\begin{array}{l}\text { Value traded to } \\
\text { GDP }\end{array}$ & & 1.00 & $\begin{array}{l}0.964^{* *} \\
(0.000)\end{array}$ & $\begin{array}{l}0.271 \\
(0.075)\end{array}$ & $\begin{array}{l}0.212 \\
(0.166)\end{array}$ \\
\hline 3. & Turnover Ratio & & & 1.00 & $\begin{array}{l}0.298^{*} \\
(0.049)\end{array}$ & $\begin{array}{l}0.252 \\
(0.099)\end{array}$ \\
\hline 4. & $\begin{array}{l}\text { ICAPM. } \\
\text { integration } \\
\text { (SBP-SP) }\end{array}$ & & & & 1.00 & $\begin{array}{l}0.679^{*} \\
(0.000)\end{array}$ \\
\hline 5. & $\begin{array}{l}\text { ICAPM.integration } \\
\text { (Share Prices) }\end{array}$ & & & & & 1.00 \\
\hline
\end{tabular}

$\mathrm{P}<0.01^{* *} \quad \mathrm{P}<0.05^{*}$

Results on the basis of Pearson's product moment correlation are given in Table 5. Results reveal that market capitalisation is significantly negatively correlated with Turnover ratio $(r=-0.355)$ and insignificantly negatively correlated with Value traded to GDP suggesting that market size and liquidity do not move together in Pakistan's stock market as should not be the case. Rather a tradeoff exists between the size and the liquidity indicators. This is so because where on the one hand liquidity rose rapidly due to active trading in a few scrips only, market capitalisation rose very slowly. This was due to the fact that most of the listed companies were not floating in more shares, which could have had a substantial increase in market capitalisation, just because there was no demand for their issues. The gradual increase is just attributable to the active chips.

Another important finding is that market size and liquidity on the basis of value traded to GDP do not have significant positive correlations with ICAPM market integration based on pricing errors of the SBP \& SP Index. This again speaks of the poor development of the market.

We obtain similar results if the average values of the indicators are taken in place of the end period values. 
Atif Naveed 171

\section{Linkage Between Indicators of Financial Intermediary Development}

Table 6: Correlation Amongst Indicators of Financial Intermediary Development

\begin{tabular}{|c|c|c|c|c|c|c|c|c|}
\hline & Variables & 1 & 2 & 3 & 4 & 5 & 6 & 7 \\
\hline 1. & $\begin{array}{l}\text { Liquid } \\
\text { liabilities to } \\
\text { GDP }\end{array}$ & 1.00 & $\begin{array}{l}0.348^{*} \\
(0.020)\end{array}$ & $\begin{array}{l}0.783^{* *} \\
(0.000)\end{array}$ & $\begin{array}{l}0.713^{* *} \\
(0.000)\end{array}$ & $\begin{array}{l}0.350^{*} \\
(0.020)\end{array}$ & $\begin{array}{l}0.317^{*} \\
(0.036)\end{array}$ & $\begin{array}{l}-0.361^{*} \\
(0.016)\end{array}$ \\
\hline 2. & $\begin{array}{l}\text { Pvt. Domestic } \\
\text { credit to GDP }\end{array}$ & & 1.00 & $\begin{array}{l}0.011 \\
(0.945)\end{array}$ & $\begin{array}{l}0.544^{* *} \\
(0.000)\end{array}$ & $\begin{array}{l}-0.585^{* *} \\
(0.000)\end{array}$ & $\begin{array}{l}-0.680^{* *} \\
(0.000)\end{array}$ & $\begin{array}{l}0.615^{* *} \\
(0.000)\end{array}$ \\
\hline 3. & $\begin{array}{l}\text { Total. } \\
\text { Domestic } \\
\text { credit to GDP }\end{array}$ & & & 1.00 & $\begin{array}{l}0.618^{* *} \\
(0.000)\end{array}$ & $\begin{array}{l}0.573 * * \\
(0.000)\end{array}$ & $\begin{array}{l}0.529 * * \\
(0.000)\end{array}$ & $\begin{array}{l}-0.630 * \\
(0.000)\end{array}$ \\
\hline 4. & $\begin{array}{l}\text { Quasi- Liquid } \\
\text { liabilities to } \\
\text { GDP }\end{array}$ & & & & 1.00 & $\begin{array}{l}0.094 \\
(0.545)\end{array}$ & $\begin{array}{l}-0.143 \\
(0.355)\end{array}$ & $\begin{array}{l}-0.026 \\
(0.869)\end{array}$ \\
\hline 5. & $\begin{array}{l}\text { Assets of } \\
\text { scheduled } \\
\text { banks to GDP }\end{array}$ & & & & & 1.00 & $\begin{array}{l}0.898 * * \\
(0.000)\end{array}$ & $\begin{array}{l}- \\
0.901 * * \\
(0.000)\end{array}$ \\
\hline 6. & $\begin{array}{l}\text { Assets of } \\
\text { NBFIs to GDP }\end{array}$ & & & & & & 1.00 & $\begin{array}{l}- \\
0.947^{* *} \\
(0.000)\end{array}$ \\
\hline 7. & $\begin{array}{l}\text { Amount of } \\
\text { saving } \\
\text { schemes to } \\
\text { GDP. }\end{array}$ & & & & & & & 1.00 \\
\hline
\end{tabular}

$\mathrm{P}<0.01 * * \quad \mathrm{P}<0.05 *$

The results on the basis of Pearson's product moment correlation have been displayed in Table 6 . Results reveal that the ratio of liquid liabilities to GDP has significant positive correlation with domestic credit extended to the private sector $(\mathrm{r}=0.348)$ as well as with total domestic credit to GDP ( $\mathrm{r}$ $=0.783$ ) presumably suggesting that of the received liabilities, most are extended as advances to the public and private sector to generate economic activity. This speaks of good intermediation services of Pakistan's financial sector. The liquid liabilities to GDP is significantly positively correlated with quasi-liquid liabilities to GDP $(r=0.713)$. This result suggests that increase in liquid liabilities was partially because of increase in long-term liabilities over the years, which in other words constitute the quasi-liquid liabilities. This augurs well for the bank and non-bank sector's development. The liquid liabilities to GDP has significant positive correlation with assets of scheduled banks to GDP as well $(\mathrm{r}=0.350)$. This is so because advances by the scheduled banks, which are their assets, are generated through the liquid liabilities of the financial sector. Moreover significant positive correlation also exists between 
liquid liabilities to GDP and assets of non-bank financial corporations ( $\mathrm{r}=$ 0.317 ) although the coefficient is not very high. Negative significant correlation exists between liquid liabilities and amount of national savings schemes $(r=-$ $0.361)$.

The assets of scheduled banks to GDP have a significant negative correlation with private sector credit to GDP ( $\mathrm{r}=-0.585)$ which turns to significant positive with total domestic credit to GDP $(\mathrm{r}=0.573)$. This was so because the scheduled banks are supposed to keep thirty percent deposits as liquidity requirement with the government, due to which less assets are left for advancing credit to the private sector. There is significant negative correlation observed between private sector credit to GDP and assets of NBFIs $(r=-0.680)$ while the relationship between assets of NBFIs to GDP and total credit extended to GDP is positively significant $(\mathrm{r}=0.589)$.

The quasi-liquid liabilities to GDP show insignificant positive correlation with the assets of scheduled banks $(r=0.094)$. This is suggestive of the fact that though advances were extended by the scheduled banks out of quasi-liquid liabilities yet less relatively as out of liquid liabilities. This in turn reveals an important outcome that though scheduled banks did extend loans for long term periods, presumably short term financing was relatively greater even out of long term liabilities.

The amounts of national saving schemes (NSS) to GDP have negative significant correlation $(\mathrm{r}=-0.901)$ with scheduled bank assets to GDP as well as with assets of NBFIs to GDP. In fact over the years, the rates of return on NSS had been pegged higher than the deposit rates of the banks which have caused difficulty in deposit mobilisation to the banks and in turn hindered their asset formation.

These results are obtained using the average values of the indicators. Similar results are found using end period values of the indicators. 
Atif Naveed 173

\section{Relationship Between Aggregate Indexes of Stock Market Development}

Table 7: Correlation Amongst Indexes of Stock Market Development

\begin{tabular}{lllll}
\hline & Variables & 1 & 2 & 3 \\
\hline 1. & INDEX 1 & 1.00 & $0.934^{* * *}$ & $0.951^{* *}$ \\
& & & $(0.000)$ & $(0.000)$ \\
2. INDEX 2 & & 1.00 & $0.961^{* *}$ \\
& & & $(0.000)$ \\
$3 . \quad$ INDEX 3 & & & 1.00 \\
\hline $\mathrm{P}<0.01^{* *}$ & & &
\end{tabular}

$\mathrm{P}<0.01 * *$

The correlation results obtained are shown in Table 7. On the basis of the relationship between the means removed aggregate indexes of stock market development, it is found that INDEX 1 has strong positive correlation with INDEX $2(\mathrm{r}=0.934)$ and INDEX $3(\mathrm{r}=0.951)$. However, this is so because the three indexes have almost identical indicators except for indicators of market integration and therefore grow together over time. Thus they lack economic significance. Identical results are obtained when the indexes are constructed using the average values of the indicators.

\section{Relationship Between Aggregate Indexes of Financial Intermediary}

Development

Table 8: Correlation Amongst Indexes of Financial Intermediary Development

\begin{tabular}{lllll}
\hline & Variables & 1 & 2 & 3 \\
\hline 1. & INDEX 1 & 1.00 & $0.406^{* *}$ & $0.741^{* *}$ \\
& & & $(0.006)$ & $(0.000)$ \\
2. & INDEX 2 & 1.00 & $0.736^{* *}$ \\
& & & $(0.000)$ \\
3. & INDEX 3 & & 1.00 \\
\hline \multicolumn{2}{l}{$\mathrm{P}<0.01^{* *}$} & & &
\end{tabular}

The correlation results are displayed in Table 8 . Unlike the stock market indexes, the financial intermediary indexes are not identical and therefore their correlations hold economic significance. On the basis of the means removed indexes, significant positive relationship prevails between all indexes of the financial intermediary development. This positive result 
suggests that there has been integrated development in all the financial intermediary sectors over time. Identical results are obtained when the indexes are constructed using the end period values of the indicators instead of average values.

Linkage Between Indicators of Stock Market Development and Financial Intermediary Development

Table 9: Correlation Between Indicators of Stock Market and Financial Intermediary Development

\begin{tabular}{|c|c|c|c|c|c|c|c|c|}
\hline & Variables & $\begin{array}{l}\text { Liquid } \\
\text { liabilities } \\
\text { to GDP }\end{array}$ & $\begin{array}{l}\text { Pvt. } \\
\text { Sector } \\
\text { credit } \\
\text { to } \\
\text { GDP }\end{array}$ & $\begin{array}{l}\text { Total } \\
\text { credit } \\
\text { to } \\
\text { GDP }\end{array}$ & $\begin{array}{l}\text { Quasi } \\
\text { liquid } \\
\text { to } \\
\text { GDP }\end{array}$ & $\begin{array}{l}\text { Assets } \\
\text { of } \\
\text { banks } \\
\text { to GDP }\end{array}$ & $\begin{array}{l}\text { Assets } \\
\text { of } \\
\text { NBFCs } \\
\text { to GDP }\end{array}$ & $\begin{array}{l}\text { Amount } \\
\text { Sav. } \\
\text { schemes } \\
\text { to GDP }\end{array}$ \\
\hline 1. & $\begin{array}{l}\text { Market Cap } \\
\text { to GDP }\end{array}$ & $\begin{array}{l}0.603^{* *} \\
(0.000)\end{array}$ & $\begin{array}{l}-0.169 \\
(0.272)\end{array}$ & $\begin{array}{l}0.630^{*} \\
* \\
(0.000)\end{array}$ & $\begin{array}{l}0.431^{* *} \\
(0.003)\end{array}$ & $\begin{array}{l}0.688^{* *} \\
(0.000)\end{array}$ & $\begin{array}{l}0.642^{* *} \\
(0.000)\end{array}$ & $\begin{array}{l}-0.615^{\text {*** }} \\
(0.000)\end{array}$ \\
\hline 2. & $\begin{array}{l}\text { Value } \\
\text {.traded to } \\
\text { GDP }\end{array}$ & $\begin{array}{l}-0.070 \\
(0.650)\end{array}$ & $\begin{array}{l}0.696^{* *} \\
(0.000)\end{array}$ & $\begin{array}{l}-0.308^{*} \\
(0.042)\end{array}$ & $\begin{array}{l}0.242 \\
(0.114)\end{array}$ & $\begin{array}{l}-0.699 * * \\
(0.000)\end{array}$ & $\begin{array}{l}-0.750^{* * *} \\
(0.000)\end{array}$ & $\begin{array}{l}0.792^{* *} \\
(0.000)\end{array}$ \\
\hline 3. & $\begin{array}{l}\text { Turnover } \\
\text { ratio }\end{array}$ & $\begin{array}{l}-0.117 \\
(0.448)\end{array}$ & $\begin{array}{l}0.744^{* *} \\
(0.000)\end{array}$ & $\begin{array}{l}-0.370^{*} \\
(0.014)\end{array}$ & $\begin{array}{l}0.190 \\
(0.216)\end{array}$ & $\begin{array}{l}-0.822^{* *} \\
(0.000)\end{array}$ & $\begin{array}{l}-0.834^{* *} \\
(0.000)\end{array}$ & $\begin{array}{l}0.867^{* *} \\
(0.000)\end{array}$ \\
\hline 4. & $\begin{array}{l}\text { ICAPM } \\
\text {.integration } \\
\text { (SBP-SP) }\end{array}$ & $\begin{array}{l}0.064 \\
(0.682)\end{array}$ & $\begin{array}{l}0.286 \\
(0.060)\end{array}$ & $\begin{array}{l}0.055 \\
(0.720)\end{array}$ & $\begin{array}{l}0.299 * * \\
(0.048)\end{array}$ & $\begin{array}{l}-0.190 \\
(0.218)\end{array}$ & $\begin{array}{l}-0.337^{* *} \\
(0.025)\end{array}$ & $\begin{array}{l}0.180 \\
(0.243)\end{array}$ \\
\hline 5. & $\begin{array}{l}\text { ICAPM.inte } \\
\text { gration } \\
\text { (S. Prices) }\end{array}$ & $\begin{array}{l}0.016 \\
(0.920)\end{array}$ & $\begin{array}{l}0.303^{*} \\
(0.046)\end{array}$ & $\begin{array}{l}-0.121 \\
(0.434)\end{array}$ & $\begin{array}{l}0.285 \\
(0.061)\end{array}$ & $\begin{array}{l}-0.148 \\
(0.337)\end{array}$ & $\begin{array}{l}-0.316^{*} \\
(0.037)\end{array}$ & $\begin{array}{l}0.170 \\
(0.269)\end{array}$ \\
\hline
\end{tabular}

$\mathrm{P}<0.01^{* *} \quad \mathrm{P}<0.05^{*}$

Correlation results to ascertain if stock market development in Pakistan is linked to the financial intermediary development are given in Table 9. Analysis of the results reveals that market capitalisation to GDP is significantly correlated with liquid liabilities to GDP $(\mathrm{r}=0.603)$, total domestic credit to GDP ( $\mathrm{r}=0.630)$, Quasi-liquid liabilities to GDP ( $\mathrm{r}=$ $0.431)$, assets of scheduled banks to GDP $(r=0.688)$ and the assets of NBFCs to GDP $(r=0.692)$. This reflects a positive trend that over the years market capitalisation to GDP which is a genuine reflector of Pakistan's stock market development has remained linked with most of the indicators of financial intermediary development.

Market liquidity is negatively significantly correlated with most of the financial intermediary sector's indicators. Value traded to GDP is negatively significantly correlated with total domestic credit to GDP ( $\mathrm{r}=$ $0.308)$, assets of scheduled banks to GDP $(r=-0.699)$ and assets of NBFCs 
to GDP $(r=-0.750)$. Similarly, turnover ratio is negatively significantly correlated with total domestic credit to GDP $(r=-0.370)$, assets of scheduled banks to GDP $(\mathrm{r}=-0.822)$ and assets of NBFCs to GDP $(\mathrm{r}=$ 0.834). Since, NSS amounts to GDP have in general contributed to financial repression in Pakistan, significant positive correlation between it and the liquidity indicators does not in a true sense reflect co-development. Moreover, the assets of NBFCs to GDP are negatively significantly correlated with both the indicators of ICAPM pricing errors while other financial intermediary sectors' indicators lack significant correlation with the indicators of market integration.

Thus, on the basis of these results it may be concluded that the stock market and the financial intermediary sector did not co-develop over the period of the study, rather a significant trade-off existed between the two. The only positive significant result was relatively strong linkage between the indicator of financial sector and market capitalisation to GDP.

These results have been obtained using the average values of the indicators. Similar results were found using end period values of the indicators.

\section{Linkage Between Aggregate Indexes of Stock Market Development and Financial Intermediary Development}

Table 10: Correlation Between Indexes and Findexes

\begin{tabular}{llcll}
\hline & Variables & FINDEX 1 & FINDEX 2 & FINDEX 3 \\
\hline 1. & INDEX 1 & -0.203 & $-0.610^{* *}$ & $-0.301^{*}$ \\
& & $(0.185)$ & $(0.000)$ & $(0.047)$ \\
2. & INDEX 2 & -0.136 & $-0.635^{* *}$ & $-0.368^{*}$ \\
& & $(0.379)$ & $(0.000)$ & $(0.014)$ \\
3. & INDEX 3 & -0.198 & $-0.628^{* *}$ & $-0.383^{*}$ \\
& & $(0.198)$ & $(0.000)$ & $(0.010)$ \\
\hline
\end{tabular}

$\mathrm{P}<0.01^{* *}$

$\mathrm{P}<0.05^{*}$

The findings derived above emerge still more strongly if we compare the link between aggregate indexes of the two sectors (Table 10). Results show negative significant correlations between INDEXES of stock market and FINDEX 2 and FINDEX 3 of financial intermediary sector while insignificant positive correlation is observed between FINDEX 1 of financial sector and the indexes of the stock market. 
These results are obtained using the end period values of the indicators. Similar results are found if indexes are constructed using the average values of the indicators.

\section{Conclusion}

The correlation results clearly depict that the stock market of Pakistan has lacked integrated development over the period of study. Unlike the stock market, the financial intermediary sector is relatively well developed and has experienced integrated development. Moreover, a debtequity trade-off emerges in the financial sector of Pakistan, which needs to be eliminated for the financial sector to become more efficient and contribute more to the growth of the economy. 
Atif Naveed 177

\section{References}

Hussain Fazal and Muhammad Ali Qasim, The Pakistani Equity Market in 50 Years : A Review, The Pakistan Development Review, Vol. (4), 1997.

Hussain Fazal and Uppal, Random Walk Model on Karachi Stock Exchange, The Pakistan Development Review, Vo1. (4) 1994.

Khilji. N, The Behaviour of Stock Returns in an Emerging Market : A Case Study of Pakistan, The Pakistan Development Review, Vol. (4), 1993.

King. Robert.G.and Ross Levine, Finance and Growth Schumpeter might be right. Quarterly Journal of Economics, Vo1. (108), 1993.

King. Robert.G.and Ross Levine, Finance, Entrepreneurship and Growth : Theory and Evidence. Journal of Monetary Economics, Vo1. (3), 1993.

Kunt Demirgue and Vojislav Maksinovic, Stock Market Development and Financing Choices of Firms, The World Bank Economic Review, Vol. (2) 1996.

Kunt Demirgue and Ross Levine, Stock Market Development and Financial intermediaries: Stylized facts, The World Bank Economic Review, Vol. (2) 1996.

Levine Ross and Sara Zervos, Stock Market Development and Economic Growth, The World Bank Economic Review, Vol. (2), 1996.

Levine, Ross. Financial development and economic growth: Views and agenda, Journal of Economic Literature, Vo1 (35), 1997.

Levine Ross and Sara Zervos, Stock Markets, Banks and Economic Growth, The American Economic Review, Vo1. (88), 1998.

Rajan, Raghuram G. and Luigi Zingales, financial dependence and growth, The American Economic Review, Vol. (88), 1998.

Shah. Salman, 1992. Capital Market Development in Pakistan, (ed) LUMS. 